Commun. Fac. Sci. Univ. Ank. Ser. A1 Math. Stat.

Volume 68, Number 2, Pages 2216-2237(2019)

DOI: $10.31801 /$ cfsuasmas. 423277

ISSN 1303-5991 E-ISSN 2618-6470

http://communications.science.ankara.edu.tr/index.php?series=A1

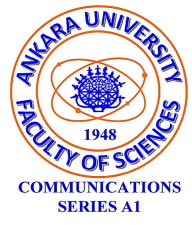

\title{
ALGEBRAIC STRUCTURE OF SQUARE MATRICES OVER RESIDUATED LATTICES
}

\author{
S. BALAEI, E. ESLAMI, AND A. BORUMAND SAEID
}

\begin{abstract}
In this paper, we introduce the algebra $\mathbb{M}_{n \times n}(L)$ of square matrices over residuated lattice $L$. The operations are induced by the corresponding operations of $L$. It is shown that the defined algebra behaves like a residuated lattice, but there are some slight differences. The properties of this algebra with respect to special residuated lattices are investigated. The notions of filter and ideal together with their roles are specified.
\end{abstract}

\section{INTRODUCTION}

Matrix theory plays an important role in various areas of science and engineering to represent different types of binary relations. For instance, in formal concept analysis which is initially developed by Wille in the 1980s [15. Formal concept analysis is a data analysis method that extracts certain concepts from the data describing the relationship between a given set of objects and a given set of attributes that the objects may or may not have [6].

In applications of formal concept analysis, the relationship between the objects and the attributes is often not binary; zero or one, but rather that there is only an extent to which the object has the attribute. Because of existence of such vague and uncertain data in real world domains like problems in economics, engineering, social science, medical science, etc., we need some tools to model these uncertain information. Since these kind of data have very important applications and their amount is enormously increasing, research on effective and efficient techniques for handling uncertain data has attracted much interest in recent years. In fact, such concerns have led some researches to reinterpret the theory of formal concepts in many-valued logics 3 . These type of problems are initially solved by $[0,1]$-valued fuzzy logic. The fuzzy matrices have been proposed to represent fuzzy relations. Thomason introduced fuzzy matrices and discussed about the convergence of the

Received by the editors: May 14, 2018; Accepted: May 02, 2019.

2010 Mathematics Subject Classification. Primary 15A30; Secondary 15B99, 15B22.

Key words and phrases. Residuated lattices, matrices over residuated lattices, operations on matrices, filter, ideal.

(C)2019 Ankara University Communications Faculty of Sciences University of Ankara-Series A1 Mathematics and Statistics 
powers of a fuzzy matrix [13. The theory of fuzzy matrices were developed by Kim and Ruosh as an extension of Boolean matrices [8]. Because of some limitations in dealing with uncertainties by fuzzy sets, Atanassov introduced theory of intuitionistic fuzzy sets as a generalization of fuzzy sets [2. Pal introduced the intuitionistic fuzzy determinant 9. Using the idea of intuitionistic fuzzy sets, Pal, Khan and Shyamal introduced the intuitionistic fuzzy matrices and studied several properties of it 10. S. Sriram and P. Murugadas worked on semiring of intuitionistic fuzzy matrices [12. A. K. Adak, et al. considered some properties of generalized intuitionistic fuzzy nilpotent matrices over distributivel lattices [1. Xiao, et al. introduced a new kind of intuitionistic fuzzy implication [16]. Moreover, motivated by results of linear algebra over fields, rings and tropical semirings, Wilding presented a systematic way to understand the behavior of matrices with entries in an arbitrary semiring [14. He focuses on three closely related problems concerning the row and column spaces of matrices. In his Ph.D. thesis, he established several new results about exactness of semirings. He showed that every Boolean ring is exact . Finally, he considers exactness for residuated lattices, showing that every involutive residuated lattice is exact [14].

Residuated structures are algebraic models of substructural logics 5]. These logics include many non-classical logics such as fuzzy logic, linear logic, relevant logic, many valued logics, intuitionistic logic and Lukasiewicz logic, where the last two are modelled by the classes of Heyting algebras and MV-algebras respectively. Therefore to reinterpret the theory of formal concepts in many- valued logics, it is natural and logical to work with matrices over residuated lattices. The papers on matrices with entries in lattices (fuzzy, intuitionistic fuzzy, residuated) do not mention the algebraic structure of the collection of corresponding matrices. This highly motivates us to consider the set of all square matrices with entries in a residuated lattice. Some of the results on square matrices can be generalized to arbitrary matrices, but since our initial goal is finding an inverse to solve systems of $\mathrm{n}$ equations with $\mathrm{n}$ unknowns, we restrict our paper to square matrices. This paper is organized as follows.

In section 2 some definitions and theorems that we need in the remaining, are given. In section 3 , all the required operations on $\mathbb{M}_{n \times n}(L)$ are defined. Properties of these operations are proved in some propositions, lemmas and theorems. In section 4, we investigate more properties of $\mathbb{M}_{n \times n}(L)$ and establish some connections between the properties of the residuated lattice $L$ and the corresponding properties of the algebra $\mathbb{M}_{n \times n}(L)$. Finally we end up with conclusion part in which our goal for the coming paper is given.

\section{Preliminaries}

In this section, we recall the definition of a residuated lattice and some basic properties that we need in the sequel. 
Definition 1. 4 An algebra $L=(L, \vee, \wedge, \odot, \rightarrow, \rightsquigarrow, 0,1)$ of type $(2,2,2,2,2,0,0)$; is a residuated lattice if it satisfies the following conditions:

(R1): $(L, \vee, \wedge, 0,1)$ is a bounded lattice,

(R2): $(L, \odot, 1)$ is a monoid,

(R3): for all $x, y, z \in L$ we have

$x \odot y \leqslant z \Leftrightarrow x \leqslant y \rightarrow z \Leftrightarrow y \leq x \rightsquigarrow z$.

$L$ is called commutative if the operation $\odot$ is commutative. In this case $x \rightarrow$ $y=x \rightsquigarrow y$, for all $x, y \in L$. Given $x \in L, x^{-}=x \rightarrow 0$ and $x^{\sim}=x \rightsquigarrow 0$. A residuated lattice $L$ is called complete if for every $S \subseteq L, \bigvee S$ and $\bigwedge S$ exist, where $\bigvee S$ and $\bigwedge S$ mean the least upper bound (sup) and greatest lower bound (inf) of $\mathrm{S}$, respectively.

We give some properties of a residuated lattice $L$ in the following theorem from [4] and [7.

Theorem 2. Any residuated lattice $L$ satisfies the following properties: For all $x, y, z \in L$

1) $x \rightarrow(y \rightarrow z)=(x \odot y) \rightarrow z$,

2) $x \rightsquigarrow(y \rightsquigarrow z)=(y \odot x) \rightsquigarrow z$,

3) $x \leq y$ iff $x \rightarrow y=1$ iff $x \rightsquigarrow y=1$,

4) $x \rightarrow x=x \rightsquigarrow x=1$,

5) $x \rightarrow 1=x \rightsquigarrow 1=1$,

6) $0 \rightarrow x=0 \rightsquigarrow x=1$,

7) $x \odot 0=0 \odot x=0$,

8) $x \odot y \leq x \wedge y$,

9) $(x \rightarrow y) \odot x \leq y$ and $x \odot(x \rightsquigarrow y) \leq y$,

10) $x \leq y \rightarrow(x \odot y)$ and $x \leq y \rightsquigarrow(y \odot x)$,

11) $x \leq y$ implies $x \odot z \leq y \odot z$ and $z \odot x \leq z \odot y$,

12) $(x \rightarrow y) \odot x \leq x \wedge y$ and $(x \rightsquigarrow y) \odot x \leq x \wedge y$,

13) $(x \rightarrow y) \odot x \leq x \leq y \rightarrow(x \odot y)$ and $(x \rightarrow y) \odot x \leq y \leq x \rightarrow(y \odot x)$,

14) $x \odot(x \rightsquigarrow y) \leq y \leq x \rightsquigarrow(x \odot y)$ and $x \odot(x \rightsquigarrow y) \leq x \leq y \rightsquigarrow(y \odot x)$,

15) if $x \leq y$ then $z \rightarrow x \leq z \rightarrow y$ and $z \rightsquigarrow x \leq z \rightsquigarrow y$,

16) if $x \leq y$ then $y \rightarrow z \leq x \rightarrow z$ and $y \rightsquigarrow z \leq x \rightsquigarrow z$,

17) $1 \rightarrow x=x$ and $1 \rightsquigarrow x=x$,

18) $x \rightarrow y=x \rightarrow(x \wedge y)$,

19) $x \rightsquigarrow y=x \rightsquigarrow(x \wedge y)$,

20) $y \leq x \rightarrow y$ and $y \leq x \rightsquigarrow y$, then $x \odot y \leq x \rightarrow y$ and $y \odot x \leq x \rightsquigarrow y$,

21) if $x \leq y$ then $x \leq z \rightarrow y$ and $x \leq z \rightsquigarrow y$,

22) $z \odot(x \wedge y) \leq(z \odot x) \wedge(z \odot y)$,

23) $(x \wedge y) \odot z \leq(x \odot z) \wedge(y \odot z)$,

24) $x \odot x^{\sim}=x^{-} \odot x=0$ and $x \leq x^{-\sim}, x \leq x^{\sim-}$,

25) $x \odot y=0$ iff $x \leq y^{-}$and $y \odot x=0$ iff $x \leq y^{\sim}$,

26) $1^{-}=1^{\sim}=0$ and $0^{-}=0^{\sim}=1$, 
27) $x \odot x=x$ then $x \odot y=x \wedge y$,

and any complete residuated lattice $L$ satisfies the following additional properties: For all $x, y, z \in L$

28) $\left(\bigvee_{i=1}^{n} x_{i}\right) \rightarrow y=\bigwedge_{i=1}^{n}\left(x_{i} \rightarrow y\right)$,

29) $\left(\bigvee_{i=1}^{n} x_{i}\right) \rightsquigarrow y=\bigwedge_{i=1}^{n}\left(x_{i} \rightsquigarrow y\right)$,

30) $\bigwedge_{i=1}^{n}\left(y \rightarrow x_{i}\right)=y \rightarrow \bigwedge_{i=1}^{n}\left(x_{i}\right)$,

31) $\bigwedge_{i=1}^{n}\left(y \rightsquigarrow x_{i}\right)=y \rightsquigarrow \bigwedge_{i=1}^{n}\left(x_{i}\right)$,

32) $x \odot\left(\bigvee_{i \in I} y_{i}\right)=\bigvee_{i \in I}(x \odot y i)$,

33) $\left(\bigvee_{i \in I} y_{i}\right) \odot x=\bigvee_{i \in I}\left(y_{i} \odot x\right)$,

34) $x \odot \bigwedge_{i=1}^{n}\left(y_{i}\right) \leq \bigwedge_{i=1}^{n}\left(x \odot y_{i}\right)$,

35) $\left(\bigvee_{i \in I_{1}} x_{i}\right) \wedge\left(\bigvee_{j \in I_{2}} y_{j}\right)=\bigvee_{i \in I_{1}} \bigvee_{j \in I_{2}}\left(x_{i} \wedge y_{j}\right)$,

36) $\left(\bigwedge_{i \in I_{1}} x_{i}\right) \wedge\left(\bigwedge_{j \in I_{2}} y_{j}\right)=\bigwedge_{i \in I_{1}} \bigwedge_{j \in I_{2}}\left(x_{i} \wedge y_{j}\right)$.

Note that (35) and (36) are also true for finite number of index sets $I_{1}, I_{2}, \ldots, I_{n}$; by induction.

Definition 3. 4] Let $L$ be a residuated lattice. A nonempty subset $F$ of $L$ is called a filter of $L$ if the following conditions hold:

i) if $x, y \in F$, then $x \odot y \in F$,

ii) if $x \in F, y \in L$ and $x \leq y$ then $y \in F$.

A filter $F$ of $L$ is proper if $F \neq L$. Clearly, $F$ is a proper filter iff $0 \notin F$.

Definition 4. 11] Let $L$ be a residuated lattice. A subset $I$ of $L$ is called ideal if the following conditions hold:

i) $0 \in I$,

ii) if $x, y \in I$, then $x \vee y \in I$,

iii) if $x \in L, y \in I$ and $x \leq y$, then $x \in I$.

An ideal $I$ of $L$ is called proper if $I \neq L$. Clearly, $I$ is proper iff $1 \notin I$. The ideal $P$ of $L$ is called prime if for all $x, y \in L$ such that $x \wedge y \in P$ then $x \in P$ or $y \in P$.

In a commutative residuated lattice $L$ we have $x^{-}=x^{\sim}$, and the common value will be denoted by $x^{*}=x \rightarrow 0$. 
Lemma 5. 11] Let $L$ be a commutative residuated lattice. If $a, b \in L$, then 1) $a \leq a^{* *}, 1^{*}=0,0^{*}=1$,

2) $a^{* * *}=a^{*}, a \leq a^{* *} \leq a^{*} \rightarrow a$,

3) $(a \wedge b)^{*}=a^{*} \vee b^{*},(a \vee b)^{*}=a^{*} \wedge b^{*}$,

4) $(a \wedge b)^{* *}=a^{* *} \wedge b^{* *},(a \vee b)^{* *}=a^{* *} \vee b^{* *}$,

5) $(a \odot b)^{* *}=a^{* *} \odot b^{* *},(a \rightarrow b)^{* *}=a^{* *} \rightarrow b^{* *}$,

6) $a \rightarrow b^{*}=b \rightarrow a^{*}=a^{* *} \rightarrow b^{*}=(a \odot b)^{*}$.

Lemma 6. 11 Let $L=(L, \vee, \wedge, \odot, \rightarrow, 0,1)$ be a commutative residuated lattice. If $x, y \in L$ such that $x \vee y=1$, then $x \odot y=x \wedge y$.

Definition 7. 11] A lattice $(L, \vee, \wedge)$ is distributive if for every $x, y, z \in L$ we have $x \vee(y \wedge z)=(x \vee y) \wedge(x \vee z)$ or equivalently $x \wedge(y \vee z)=(x \wedge y) \vee(x \wedge z)$.

Definition 8. 11 A complete residuated lattice $L$ is called Brouwerian if a $\wedge$ $\left(\bigvee_{i} b_{i}\right)=\bigvee_{i}\left(a \wedge b_{i}\right)$, for all $a, b_{i} \in L$.

Remark 9. Let $(L, \vee, \wedge, 0,1)$ be a bounded lattice. Recall (see [1]) that an element $a \in L$ is called complemented if there is an element $b \in L$ such that $a \vee b=1$ and $a \wedge b=0$. If such element $b$ exists it is called a complement of $a$. The set of all complemented elements of $L$ is denoted by $B(L)$. Complements are not generally unique, unless the lattice is distributive.

In residuated lattices however, although the underlying lattices need not be distributive, the complements are unique [11. Also, for every commutative residuated lattice $L, B(L)$ denotes the Boolean algebra of all complemented elements in $L$.

Definition 10. 11] Let $L=(L, \vee, \wedge, \odot, \rightarrow, 0,1)$ be a commutative residuated lattice. Then we say that $L$ is Boolean if $B(L)=L$.

Definition 11. 11] A commutative residuated lattice $L$ is called a $G$-algebra if $x \odot x=x$, for all $x \in L$.

We note that in every $G$-algebra $L, x \odot y=x \wedge y$ for all $x, y \in L$.

Definition 12. 11] We say that a commutative residuated lattice $L$ is locally finite if for all $x \in L, x \neq 1$, there is $n \in \mathbb{N}$ such that $x^{n}=0$. The least such $n$ is called the order of $x$ denoted by ord $(x)$.

Proposition 13. 11] In any locally finite commutative residuated lattice L, for all $x \in L:$

1) $0<x<1$ iff $0<x^{*}<1$,

2) $x^{*}=0$ iff $x=1$,

3) $x^{*}=1$ iff $x=0$. 


\section{Matrices Over Residuated Lattices}

Throughout this paper, we assume that $L$ is a commutative residuated lattice, unless otherwise stated. In this section we consider $M_{n \times n}(L)$, the set of all $n \times n$ matrices over $L$, and define some appropriate operations on $M_{n \times n}(L)$ induced by $L$ and construct the algebra $\mathbb{M}_{n \times n}(L) . A=\left[a_{i j}\right]_{n \times n}, B=\left[b_{i j}\right]_{n \times n}, C=\left[c_{i j}\right]_{n \times n}$, etc.

Definition 14. Let $M_{n \times n}(L)=\left\{\left[a_{i j}\right]_{n \times n} \mid 1 \leq i, j \leq n, a_{i j} \in L\right\}$. Define:

(1) $\left[a_{i j}\right]_{n \times n} \sqcup\left[b_{i j}\right]_{n \times n}=\left[a_{i j} \vee b_{i j}\right]_{n \times n}$,

(2) $\left[a_{i j}\right]_{n \times n} \sqcap\left[b_{i j}\right]_{n \times n}=\left[a_{i j} \wedge b_{i j}\right]_{n \times n}$,

(3) $\left[a_{i j}\right]_{n \times n} \odot\left[b_{i j}\right]_{n \times n}=\left[c_{i j}\right]_{n \times n}$, such that $c_{i j}=\bigvee_{t=1}^{n}\left(a_{i t} \odot b_{t j}\right)$,

(4) $\left[a_{i j}\right]_{n \times n} \triangleright\left[b_{i j}\right]_{n \times n}=\left[c_{i j}\right]_{n \times n}$, such that $c_{i j}=\bigwedge_{t=1}^{n}\left(a_{j t} \rightarrow b_{i t}\right)$,

(5) $\left[a_{i j}\right]_{n \times n} \triangleleft\left[b_{i j}\right]_{n \times n}=\left[c_{i j}\right]_{n \times n}$, such that $c_{i j}=\bigwedge_{t=1}^{n}\left(a_{t i} \rightarrow b_{t j}\right)$.

Let $A, B \in M_{n \times n}(L)$. Define:

(6) $A \preceq B$ iff $A \sqcap B=A$ iff $A \sqcup B=B$ iff $a_{i j} \leq b_{i j}$ for all $1 \leq i, j \leq n$,

(7) $\perp=\left[a_{i j}\right]_{n \times n}$ where $a_{i j}=0$ for all $1 \leq i, j \leq n$,

(8) $\top=\left[a_{i j}\right]_{n \times n}$ where $a_{i j}=1$ for all $1 \leq i, j \leq n$,

(9) $I_{n}=\left[a_{i j}\right]_{n \times n}$ where $a_{i j}=1$ if $i=j$ and $a_{i j}=0$ otherwise.

Based on the above, we may consider $\left(M_{n \times n}(L), \sqcup, \sqcap, \sqcup, \triangleright, \triangleleft, \perp, \top, I_{n}\right)$ as an algebra of type $(2,2,2,2,2,0,0,0)$ and show this algebra by $\mathbb{M}_{n \times n}(L)$. We see that the relation $\preceq$ defined by $(6)$ is an order on the $\mathbb{M}_{n \times n}(L)$.

Here are some properties of this structure.

Lemma 15. Let $A, B$ and $C \in M_{n \times n}(L)$. Then the following hold:

1) $A \sqcup B=B \sqcup A$ and $A \sqcap B=B \sqcap A$.

2) $A \sqcup(B \sqcup C)=(A \sqcup B) \sqcup C$ and $A \sqcap(B \sqcap C)=(A \sqcap B) \sqcap C$.

3) $A \sqcup A=A \sqcap A=A$.

4) $A \sqcup(A \sqcap B)=A \sqcap(A \sqcup B)=A$.

5) $A \sqcap \perp=\perp$ and $A \sqcup \top=\top$.

6) $(A \sqcup B) \square C=A \boxminus(B \sqcup C)$.

7) $I_{n} \triangleright A=A \boxminus I_{n}=A$.

8) $(B \sqcup C) \square A=(B \sqcup A) \sqcup(C \sqcup A)$.

9) $A \boxminus(B \sqcup C)=(A \sqcup B) \sqcup(A \sqcup C)$. 
10) $(B \sqcap C) \boxminus A \preceq(B \boxminus A) \sqcap(C \boxminus A)$.

11) $A \boxminus(B \sqcap C) \preceq(A \boxminus B) \sqcap(A \sqcup C)$.

12) $A \boxminus \perp=\perp \boxminus A=\perp$.

Proof. We only prove 6 and 11, the others are similarly proved by using the definitions of corresponding operations. Let $A=\left[a_{i j}\right]_{n \times n}, B=\left[b_{i j}\right]_{n \times n}$ and $C=\left[c_{i j}\right]_{n \times n} \in M_{n \times n}(L)$. By definition of $\square$ and Theorem 2 for all $1 \leq i, j, \leq n$, we have

$$
\begin{aligned}
((A \odot B) \odot C)_{i j} & =\bigvee_{t=1}^{n}\left(\left(\bigvee_{t^{\prime}=1}^{n}\left(a_{i t^{\prime}} \odot b_{t^{\prime} t}\right)\right) \odot c_{t j}\right) \\
& =\bigvee_{t=1}^{n}\left(\bigvee_{t^{\prime}=1}^{n}\left(a_{i t^{\prime}} \odot b_{t^{\prime} t} \odot c_{t j}\right)\right) \\
& =\bigvee_{t^{\prime}=1}^{n}\left(a_{i t^{\prime}} \odot\left(\bigvee_{t=1}^{n}\left(b_{t^{\prime} t} \odot c_{t j}\right)\right)\right) \\
& =(A \odot(B \odot C))_{i j} .
\end{aligned}
$$

To prove (11), for all $1 \leq i, j, \leq n$

$$
\begin{aligned}
(A \odot(B \sqcap C))_{i j} & =\bigvee_{t=1}^{n}\left(a_{i t} \odot\left(b_{t j} \wedge c_{t j}\right)\right) \\
& \leq \bigvee_{t=1}^{n}\left(\left(a_{i t} \odot b_{t j}\right) \wedge\left(a_{i t} \odot c_{t j}\right)\right) \\
& =\left(\bigvee_{t=1}^{n}\left(a_{i t} \odot b_{t j}\right)\right) \wedge\left(\bigvee_{t=1}^{n}\left(a_{i t} \odot c_{t j}\right)\right) \\
& =(A \odot B)_{i j} \sqcap(A \odot C)_{i j}
\end{aligned}
$$

Corollary 16. Let $L$ and $M_{n \times n}(L)$ be as above. Then

(a) $\left(M_{n \times n}(L), \sqcup, \sqcap, \perp, \top\right)$ is a bounded lattice,

(b) $\left(M_{n \times n}(L), \odot, I_{n}\right)$ is a monoid,

(c) $(\odot, \triangleright)$ and $(\square, \triangleleft)$ are two adjoint pairs, i.e.,

$A \boxminus B \preceq C$ iff $A \preceq B \triangleright C$ iff $B \preceq A \triangleleft C$, for all $A, B$ and $C \in M_{n \times n}(L)$.

Proof. (a) $\left(M_{n \times n}(L), \sqcup, \sqcap\right)$ is a lattice by 1- 4 of Lemma 15 and boundedness follows from 5 of Lemma 15 . 
(b) $\left(M_{n \times n}(L), \square, I_{n}\right)$ is a monoid by 6 and 7 of Lemma 15

(c) let $A=\left[a_{i j}\right]_{n \times n}, B=\left[b_{i j}\right]_{n \times n}$ and $C=\left[c_{i j}\right]_{n \times n} \in M_{n \times n}(L)$. Then for all $1 \leq i, j, t \leq n$

$$
\begin{aligned}
A \boxminus B \preceq C & \Leftrightarrow(A \boxminus B)_{i j} \leq c_{i j} \\
& \Leftrightarrow \bigvee_{t=1}^{n}\left(a_{i t} \odot b_{t j}\right) \leq c_{i j} \\
& \Leftrightarrow a_{i t} \odot b_{t j} \leq c_{i j} \\
& \Leftrightarrow a_{i t} \leq b_{t j} \rightarrow c_{i j} \\
& \Leftrightarrow a_{i j} \leq b_{j t} \rightarrow c_{i t} \text { by interchanging } t \text { and } j \\
& \Leftrightarrow a_{i j} \leq \bigwedge_{t=1}^{n}\left(b_{j t} \rightarrow c_{i t}\right) \\
& \Leftrightarrow A \preceq B \triangleright C .
\end{aligned}
$$

To prove the other relation for all $1 \leq i, j, t \leq n$, we have

$$
\begin{aligned}
A \triangleright B \preceq C & \Leftrightarrow(A \boxminus B)_{i j} \leq c_{i j} \\
& \Leftrightarrow \bigvee_{t=1}^{n}\left(a_{i t} \odot b_{t j}\right) \leq c_{i j} \\
& \Leftrightarrow a_{i t} \odot b_{t j} \leq c_{i j} \\
& \Leftrightarrow b_{t j} \leq a_{i t} \rightarrow c_{i j} \\
& \Leftrightarrow b_{i j} \leq a_{t i} \rightarrow c_{t j} \text { by interchanging } t \text { and } i \\
& \Leftrightarrow b_{i j} \leq \bigwedge_{t=1}^{n}\left(a_{t i} \rightarrow c_{t j}\right) \\
& \Leftrightarrow B \preceq A \triangleleft C .
\end{aligned}
$$

It is well known that in a commutative residuated lattice $(L, \vee, \wedge, \odot, \rightarrow, 0,1)$ for all $a, b \in L, a \rightarrow b=1$ iff $a \leq b$. But in $\mathbb{M}_{n \times n}(L)$ we have the following:

Lemma 17. For every $A, B \in M_{n \times n}(L), A \preceq B$ iff $I_{n} \preceq A \triangleright B$ iff $I_{n} \preceq A \triangleleft B$.

Proof. $A \preceq B$ iff $A=A \boxminus I_{n}=I_{n} \sqcup A \preceq B$ iff $I_{n} \preceq A \triangleright B$ iff $I_{n} \preceq A \triangleleft B$, by residuation propositionerty part (c) in Corollary 16 .

Remark 18. If $A=\left(\begin{array}{ll}1 & 0 \\ 0 & 1\end{array}\right), B=\left(\begin{array}{ll}1 & 1 \\ 0 & 1\end{array}\right) \in M_{n \times n}(\{0,1\})$, then $A \triangleright B=$ $A \triangleleft B=\left(\begin{array}{ll}1 & 1 \\ 0 & 1\end{array}\right) \neq \top$ and $A \preceq B$. 
Definition 14 . Corollary 16 show that $\mathbb{M}_{n \times n}(L)$, has a residuated structure in which the monoid $\left(M_{n \times n}(L), \square, I_{n}\right)$ is not generally commutative and the identity element $I_{n}$ is different from the top element $T$ of the corresponding bounded lattice in $(a)$ of Corollary 16. We will examine some properties of $\mathbb{M}_{n \times n}(L)$.

Proposition 19. Let $A, B, C$ be elements of $M_{n \times n}(L)$ such that $A \preceq B$ and $C \preceq D$, then for every $Z \in M_{n \times n}(L)$

$$
\begin{aligned}
& (a) A \square C \preceq B \boxminus D, \\
& (b) Z \square A \preceq Z \square B, \\
& (c) Z \triangleright A \preceq Z \triangleright B \text { and } Z \triangleleft A \preceq Z \triangleleft B, \\
& (d) B \triangleright Z \preceq A \triangleright Z \text { and } B \triangleleft Z \preceq A \triangleleft Z, \\
& (e) A \preceq B \triangleright(A \square B) \text { and } A \preceq B \triangleleft(B \square A), \\
& (f) A \triangleright \top=A \triangleleft \top=\top, \\
& (j) \perp \triangleright A=\perp \triangleleft A=\top, \\
& (k) I_{n} \triangleright \perp=I_{n} \triangleleft \perp=\perp, \\
& (l) \top \triangleright I_{n}=\top \triangleleft I_{n}=\perp, \\
& (m) \top \triangleright A \preceq A \text { and } \top \triangleleft A \preceq A .
\end{aligned}
$$

Proof. All of the above can be readily obtained by using the definitions of corresponding operations. For instance, for $(a)$ since in residuated lattice $L$ we have $a_{i t} \odot c_{t j} \leq b_{i t} \odot d_{t j}$ for all $i, j, t=1,2, \ldots, n$,

$(A \boxminus C)_{i j}=\bigvee_{t=1}^{n}\left(a_{i t} \odot c_{t j}\right) \leq \bigvee_{t=1}^{n}\left(b_{i t} \odot d_{t j}\right)=(B \boxminus D)_{i j}$. Then $(A \boxminus C) \preceq(B \boxminus D)$ (c) Let $A \preceq B$. Then $a_{i t} \leq b_{i t}$ for all $1 \leq i, t \leq n$, by Theorem 2 (15) we have $z_{j t} \rightarrow a_{i t} \leq z_{j t} \rightarrow b_{i t}$. Then $\bigwedge_{t=1}^{n}\left(z_{j t} \rightarrow a_{i t}\right) \leq \bigwedge_{t=1}^{n}\left(z_{j t} \rightarrow b_{i t}\right)$. So we get $Z \triangleright A \preceq Z \triangleright B$. The relation $Z \triangleleft A \preceq Z \triangleleft B$, is proved similarly. For the last part we have $(\top \triangleright A)_{i j}=\bigwedge_{t=1}^{n}\left(1 \rightarrow a_{i t}\right)=\bigwedge_{t=1}^{n} a_{i t} \leq a_{i j}$ for all $1 \leq i, j \leq n$, then $\top \triangleright A \preceq A$. The others are similarly proved.

Let $A \in M_{n \times n}(L)$. We put $A^{-}=A \triangleright \perp$ and $A^{\sim}=A \triangleleft \perp$. Then we prove:

Proposition 20. $A^{-} \square A=\perp$ and $A \square A^{\sim}=\perp$, for every $A \in M_{n \times n}(L)$.

Proof. Let $A^{-} \boxminus A=\left[x_{i j}\right]_{n \times n}$. Then $x_{i j}=\bigvee_{t=1}^{n}\left(b_{i t} \odot a_{t j}\right)$ for all $1 \leq i, j \leq n$, where $b_{i t}=\bigwedge_{k=1}^{n}\left(a_{t k} \rightarrow 0\right)$. So $x_{i j}=\bigvee_{t=1}^{n}\left(\bigwedge_{k=1}^{n}\left(a_{t k} \rightarrow 0\right) \odot a_{t j}\right)=0$, because when $k=j,\left(a_{t j} \rightarrow 0\right) \odot a_{t j}=0$, for all $t=1, \ldots, n$. 
Corollary 21. $\perp^{-}=\perp^{\sim}=\top$ and $\top^{-}=\top^{\sim}=\perp$.

Proposition 22. The following properties hold for all $A, B, C \in M_{n \times n}(L)$.

1) $(A \triangleright B) \triangleright C=A \triangleright(B \triangleright C)$,

2) $(B \sqcup A) \triangleleft C=A \triangleleft(B \triangleleft C)$,

3) $A \preceq B \triangleright(A \boxminus B)$,

4) $A \preceq B \triangleleft(B \sqcup A)$,

5) $(A \sqcup B) \triangleright C=(A \triangleright C) \sqcap(B \triangleright C)$,

6) $(A \sqcup B) \triangleleft C=(A \triangleleft C) \sqcap(B \triangleleft C)$,

7) $(A \sqcap B) \triangleright C=(A \triangleright C) \sqcup(B \triangleright C)$,

8) $(A \sqcap B) \triangleleft C=(A \triangleleft C) \sqcup(B \triangleleft C)$,

9) $(B \triangleright C) \sqcup(A \triangleright B) \preceq A \triangleright C$,

10) $(A \triangleleft B) \sqcup(B \triangleleft C) \preceq A \triangleleft C$,

11) $A \preceq(A \triangleright \perp) \triangleleft \perp$,

12) $A \preceq(A \triangleleft \perp) \triangleright \perp$.

Proof. We prove only (1),(3),(9),(11) and (12). The others are similarly proved. To prove (1) we have: $((A \sqcup B) \triangleright C)_{i j}=\bigwedge_{t=1}^{n}\left(c_{j t}^{\prime} \rightarrow c_{i t}\right)$ such that $c_{j t}^{\prime}=\bigvee_{t^{\prime}=1}^{n}\left(a_{j t^{\prime}} \odot\right.$ $\left.b_{t^{\prime} t}\right)$. Then by Theorem 2

$$
\begin{aligned}
((A \boxminus B) \triangleright C)_{i j} & =\bigwedge_{t=1}^{n}\left(\bigvee_{t^{\prime}=1}^{n}\left(a_{j t^{\prime}} \odot b_{t^{\prime} t}\right)\right) \rightarrow c_{i t} \\
& =\bigwedge_{t=1}^{n}\left(\bigwedge_{t^{\prime}=1}^{n}\left(\left(a_{j t^{\prime}} \odot b_{t^{\prime} t}\right) \rightarrow c_{i t}\right)\right) \\
& =\bigwedge_{t=1}^{n}\left(\bigwedge_{t^{\prime}=1}^{n}\left(a_{j t^{\prime}} \rightarrow\left(b_{t^{\prime} t} \rightarrow c_{i t}\right)\right)\right) \\
& =\bigwedge_{t^{\prime}=1}^{n}\left(\bigwedge_{t=1}^{n}\left(a_{j t^{\prime}} \rightarrow\left(b_{t^{\prime} t} \rightarrow c_{i t}\right)\right)\right) \\
& =\bigwedge_{t^{\prime}=1}^{n}\left[\left(a_{j t^{\prime}} \rightarrow\left(\bigwedge_{t=1}^{n}\left(b_{t^{\prime} t} \rightarrow c_{i t}\right)\right)\right]\right. \\
& =\bigwedge_{t^{\prime}=1}^{n}\left(a_{j t^{\prime}} \rightarrow\left(b_{i t^{\prime}}\right)\right) \text { where } b_{i t^{\prime}}^{\bullet}=\bigwedge_{t=1}^{n}\left(b_{t^{\prime} t} \rightarrow c_{i t}\right) \\
& =(A \triangleright(B \triangleright C))_{i j} .
\end{aligned}
$$

Then $(A \triangleright B) \triangleright C=A \triangleright(B \triangleright C)$.

To prove (3), we have

$$
((A \sqcup B) \triangleright C)_{i j}=\left(\bigwedge_{t=1}^{n} c_{j t}^{\bullet} \rightarrow c_{i t}\right)
$$




$$
\begin{aligned}
= & \left(\bigwedge_{t=1}^{n}\left(a_{j t} \vee b_{j t}\right) \rightarrow c_{i t}\right) \text { where } c_{j t}^{\bullet}=\left(a_{j t} \vee b_{j t}\right) \\
= & \left(\bigwedge_{t=1}^{n}\left[\left(a_{j t} \rightarrow c_{i t}\right) \wedge\left(b_{j t} \rightarrow c_{i t}\right)\right]\right) \\
= & {\left[\bigwedge_{t=1}^{n}\left[\left(a_{j t} \rightarrow c_{i t}\right)\right] \wedge\left[\bigwedge_{t=1}^{k}\left(b_{j t} \rightarrow c_{i t}\right)\right]\right] } \\
= & (A \triangleright C)_{i j} \sqcap(B \triangleright C)_{i j} .
\end{aligned}
$$

Then $(A \sqcup B) \triangleright C=(A \triangleright C) \sqcap(B \triangleright C)$. For (9) take $D=A \triangleright B$. Then in particular $D \preceq A \triangleright B$ so $D \square A \preceq B$. Similarly, if we take $D^{\prime}=B \triangleright C$, then we have $D^{\prime} \boxminus B \preceq C$, and thus $D^{\prime} \bullet(D \boxminus A)=\left(D^{\prime} \square D\right) \square A \preceq D^{\prime} \boxminus B \preceq C$. Therefore $D^{\prime} \unlhd D \preceq A \triangleright C$, which means that $(B \triangleright C) \square(A \triangleright B) \preceq A \triangleright C$. The proof of (10) is similar. (11) and (12) follows from Proposition 20.

Theorem 23. If $L$ is a complete residuated lattice, $A \in M_{n \times n}(L)$ and $\left(B_{i}\right)_{i \in I}$ is a family of elements of $M_{n \times n}(L)$, then

1) $A \sqcup\left(\sqcup_{i \in I} B_{i}\right)=\sqcup_{i \in I}\left(A \sqcup B_{i}\right)$,

2) $\left(\sqcup_{i \in I} B_{i}\right) \triangleright A=\sqcup_{i \in I}\left(B_{i} \boxminus A\right)$,

3) $A \boxminus\left(\sqcap_{i \in I} B_{i}\right) \preceq \sqcap_{i \in I}\left(A \triangleright B_{i}\right)$,

4) $\left(\sqcap_{i \in I} B_{i}\right) \triangleright A \preceq \sqcap_{i \in I}\left(B_{i} \odot A\right)$,

5) $A \triangleright\left(\sqcap_{i \in I} B_{i}\right) \preceq \sqcap_{i \in I}\left(A \triangleright B_{i}\right)$,

6) $A \triangleleft\left(\sqcap_{i \in I} B_{i}\right) \preceq \sqcap_{i \in I}\left(A \triangleleft B_{i}\right)$,

7) $\left(\sqcup_{i \in I} B_{i}\right) \triangleright A \preceq \sqcap_{i \in I}\left(B_{i} \triangleright A\right)$,

8) $\left(\sqcup_{i \in I} B_{i}\right) \triangleleft A \preceq \sqcap_{i \in I}\left(B_{i} \triangleleft A\right)$.

Proof. We prove only (1),(5) and (7). The others are similarly proved. For (1) Clearly, $A \boxminus B_{i} \preceq A \sqcup\left(\sqcup_{i \in I} B_{i}\right)$, for each $i \in I$. Therefore $\sqcup_{i \in I}\left(A \sqcup B_{i}\right) \preceq A \sqcup$ $\left(\sqcup_{i \in I} B_{i}\right)$. Conversely, since for every $i \in I, A \sqcup B_{i} \preceq \sqcup_{i \in I}\left(A \sqcup B_{i}\right), B_{i} \preceq A \triangleleft$ $\left[\sqcup_{i \in I}\left(A \sqcup B_{i}\right)\right]$. So $\sqcup_{i \in I} B_{i} \preceq A \triangleleft\left[\sqcup_{i \in I}\left(A \sqcup B_{i}\right)\right]$. Therefore $A \sqcup\left(\sqcup_{i \in I} B_{i}\right) \preceq$ $\sqcup_{i \in I}\left(A \sqcup B_{i}\right)$, and we obtain the requested equality.

To prove (5) it is enough to set $\Pi_{i \in I} B_{i}=B$. Then $B \preceq B_{i}$ for each $i \in I$. So $A \triangleright B \preceq A \triangleright B_{i}$ and we have $A \triangleright\left(\sqcap_{i \in I} B_{i}\right) \preceq \sqcap_{i \in I}\left(A \triangleright B_{i}\right)$. For $(7)$ put 
$B=\sqcup_{i \in I} B_{i}$, then $B_{i} \preceq B$ for every $i \in I$, so $B \triangleright A \preceq B_{i} \triangleright A$. Therefore $\left(\sqcup_{i \in I} B_{i}\right) \triangleright A \preceq \sqcap_{i \in I}\left(B_{i} \triangleright A\right)$.

Proposition 24. Let $A=\left[a_{i j}\right]_{n \times n}, B=\left[b_{i j}\right]_{n \times n} \in M_{n \times n}(L)$. If $A \sqcup B=\top$, then $A \sqcap B=\left[c_{i j}\right]_{n \times n}=\left[a_{i j} \odot b_{i j}\right]_{n \times n}$, for all $1 \leq i, j \leq n$.

Proof. If $A \sqcup B=\top$, then $a_{i j} \vee b_{i j}=1$ for $1 \leq i, j \leq n$. By Lemma 6 we have $a_{i j} \wedge$ $b_{i j}=a_{i j} \odot b_{i j}$ for $1 \leq i, j \leq n$. Therefore $A \sqcap B=\left[a_{i j} \wedge b_{i j}\right]_{n \times n}=\left[a_{i j} \odot b_{i j}\right]_{n \times n}$.

We note that if $L$ is a G-algebra, we have always $A \sqcap B=\left[a_{i j} \odot b_{i j}\right]_{n \times n}$.

We showed that $\mathbb{M}_{n \times n}(L)$ behaves like a residuated lattice. But there are some differences. There are some properties of residuated lattices which do not hold in $\mathbb{M}_{n \times n}(L)$.

Example 25. Let $L=\{0, a, b, 1\}$ with $0<a, b<1$ such that $a, b$ are incomparable. Then $L$ is a commutative residuated lattice relative to the following operations

\begin{tabular}{|c|c|c|c|c|c|c|c|c|c|}
\hline$\odot$ & 0 & $a$ & $b$ & 1 & $\rightarrow$ & 0 & $a$ & $b$ & 1 \\
\hline 0 & 0 & 0 & 0 & 0 & 0 & 1 & 1 & 1 & 1 \\
\hline$a$ & 0 & $a$ & 0 & $a$ & $a$ & $b$ & 1 & $b$ & 1 \\
\hline$b$ & 0 & 0 & $b$ & $b$ & $b$ & $a$ & $a$ & 1 & 1 \\
\hline 1 & 0 & $a$ & $b$ & 1 & 1 & 0 & $a$ & $b$ & 1 \\
\hline
\end{tabular}

by counter examples we show that the following properties do not hold in the algebra $\mathbb{M}_{n \times n}(L)$, for every $A, B, C \in M_{n \times n}(L)$.

1) $A \triangleright A=\top$,

2) $A \boxminus B \preceq A \sqcap B$,

3) $\top \triangleright A=A$,

4) if $A \preceq B$ then $A \preceq C \triangleright B$, in particular $A \preceq C \triangleright A$,

5) $A \triangleright B \preceq A \triangleright B$,

6) if $A \boxminus A=A$ then $A \boxminus B=A \sqcap B$,

7) $A \boxminus(A \triangleright B) \preceq B$,

8) $(A \triangleright B)=A \triangleright(A \sqcap B)$,

9) $B \preceq A \triangleright B$. 
Proof. We get these matrices of $M_{2 \times 2}(L)$. (1) if $A=\left(\begin{array}{ll}1 & 0 \\ 0 & 1\end{array}\right)$. Then $A \triangleright A=$ $I_{2} \neq \top$.

(2) if $A=\left(\begin{array}{ll}a & b \\ 0 & 0\end{array}\right)$ and $B=\left(\begin{array}{cc}a & 1 \\ 0 & b\end{array}\right)$. Then $A \boxminus B=\left(\begin{array}{cc}a & a \vee b \\ 0 & 0\end{array}\right) \npreceq A \sqcap B=$ $\left(\begin{array}{ll}a & b \\ 0 & 0\end{array}\right)=A$.

(3) put $A=I_{2}$, then $\top \triangleright I_{2}=\perp$ and $I_{2} \neq \perp$.

(4) let $A=\left(\begin{array}{ll}a & b \\ 0 & 0\end{array}\right)$ and $B=\left(\begin{array}{ll}a & 1 \\ 0 & b\end{array}\right)$ and $C=\left(\begin{array}{ll}b & 1 \\ 1 & a\end{array}\right)$. Then $C \triangleright B=$ $\left(\begin{array}{ll}a & a \\ 0 & 0\end{array}\right)$ we get $A \npreceq C \triangleright B$, and $C \triangleright A=\perp$ then $A \npreceq \perp$.

(5) put $A=\left(\begin{array}{ll}1 & 1 \\ 0 & 0\end{array}\right)$ and $B=\left(\begin{array}{ll}0 & 0 \\ 1 & 1\end{array}\right)$ then $A \boxminus B=\left(\begin{array}{ll}1 & 1 \\ 0 & 0\end{array}\right)$ and $A \triangleright B=$ $\left(\begin{array}{ll}0 & 1 \\ 1 & 1\end{array}\right)$, so $A \boxminus B \npreceq A \triangleright B$.

(6) set $A=I_{2}$ and $B=\left(\begin{array}{ll}0 & 1 \\ 1 & 0\end{array}\right)$ then $A \square A=A$ and $A \boxminus B=B \neq A \sqcap B=\perp$.

(7) let $A=\left(\begin{array}{ll}1 & 1 \\ 0 & 0\end{array}\right)$ and $B=\left(\begin{array}{ll}0 & 0 \\ 1 & 1\end{array}\right)$. Then $A \triangleright B=\left(\begin{array}{cc}0 & 1 \\ 1 & 1\end{array}\right)$ and $A \triangleright(A \triangleright$ $B)=\left(\begin{array}{ll}1 & 1 \\ 0 & 0\end{array}\right) \npreceq B=\left(\begin{array}{ll}0 & 0 \\ 1 & 1\end{array}\right)$.

(8) let $A=\left(\begin{array}{ll}1 & 0 \\ 0 & 1\end{array}\right)$ and $B=\left(\begin{array}{ll}0 & 1 \\ 1 & 0\end{array}\right)$. Then $A \triangleright B=\left(\begin{array}{ll}0 & 1 \\ 1 & 0\end{array}\right)$ and $A \triangleright(A \sqcap$ $B)=\left(\begin{array}{ll}0 & 0 \\ 0 & 0\end{array}\right)$.

(9) put $B=I_{2}, A=\top$ then $\top \triangleright I_{2}=\perp$ and $I_{2} \npreceq \perp$.

Remark 26. The results that we have proved so far for commutative residuated lattices can be extended to generalized residuated lattices, i.e., not necessarily commutative ones (Definition 1). In this case the operations $\triangleright$ and $\triangleleft$ are defined in terms of $\rightarrow$ and $\rightsquigarrow$ respectively.

Now, we show that the basic Corollary 16 is extended to matrices over generalized residuated lattices.

The results of Corollary 16 can be extended to matrices over generalized residuated lattices as follows:

Theorem 27. Let $L$ be a generalized residuated lattice and $M_{n \times n}(L)$ be the set of all square matrices over $L$. Then

(a) $\left(M_{n \times n}(L), \sqcup, \sqcap, \perp, \top\right)$ is a bounded lattice,

(b) $\left(M_{n \times n}(L), \odot, I_{n}\right)$ is a monoid, 
(c) $(\square, \triangleright)$ and $(\square, \triangleleft)$ are two adjoint pairs, i.e.,

$A \sqcup B \preceq C$ iff $A \preceq B \triangleright C$ iff $B \preceq A \triangleleft C$, for all $A, B$ and $C \in M_{n \times n}(L)$.

Proof. Let $\left[a_{i j}\right]_{n \times n},\left[b_{i j}\right]_{n \times n},\left[c_{i j}\right]_{n \times n} \in M_{n \times n}(L)$. It is enough like the previous case $\left[a_{i j}\right]_{n \times n} \triangleright\left[b_{i j}\right]_{n \times n}=\left[c_{i j}\right]_{n \times n}$, such that $c_{i j}=\bigwedge_{t=1}^{n}\left(a_{j t} \rightarrow b_{i t}\right)$ and define: $\left[a_{i j}\right]_{n \times n} \triangleleft\left[b_{i j}\right]_{n \times n}=\left[c_{i j}\right]_{n \times n}$, such that $c_{i j}=\bigwedge_{t=1}^{n}\left(a_{t i} \rightsquigarrow b_{t j}\right)$. The proof is similar to Corollary 16

\section{Some more properties of $\mathbb{M}_{n \times n}(L)$}

In this section, we investigate more properties of $\mathbb{M}_{n \times n}(L)$. In the following we are going to establish some connections between the properties of the residuated lattice $L$ and the corresponding properties of the $\mathbb{M}_{n \times n}(L)$.

Proposition 28. $\mathbb{M}_{n \times n}(L)$ is distributive iff $L$ distributive.

Proof. Let $A=\left[a_{i j}\right]_{n \times n}, B=\left[b_{i j}\right]_{n \times n}, C=\left[c_{i j}\right]_{n \times n} \in M_{n \times n}(L)$. Then the result follows from the following identities:

$$
\begin{aligned}
(A \sqcap(B \sqcup C))_{i j} & =a_{i j} \wedge\left(b_{i j} \vee c_{i j}\right) \\
& =\left(a_{i j} \wedge b_{i j}\right) \vee\left(a_{i j} \wedge c_{i j}\right) \\
& =(A \sqcap B)_{i j} \vee(A \sqcap C)_{i j} \\
& =((A \sqcap B) \sqcup(A \sqcap C))_{i j} .
\end{aligned}
$$

Definition 29. The element $A \in M_{n \times n}(L)$ is called complemented if there is an element $B \in M_{n \times n}(L)$ such that $A \sqcup B=\top$ and $A \sqcap B=\perp$. Such an element $B$ is called a complement element of $A$.

The following proposition together with Remark 9, show that if $L$ is complemented, then every $A \in M_{n \times n}(L)$ has a unique complement $B$ denoted by $B=A^{c}$. The set of all complemented elements of $M_{n \times n}(L)$ is denoted by $B\left(M_{n \times n}(L)\right)$.

Proposition 30. $L$ is complemented iff $\mathbb{M}_{n \times n}(L)$ is complemented.

Proof. Let $A=\left[a_{i j}\right]_{n \times n} \in M_{n \times n}(L)$. Then $a_{i j} \in L$ for all $i, j=1,2,3, \ldots, n$. Since $L$ is complemented, there is unique $b_{i j} \in L$ such that $a_{i j} \vee b_{i j}=1$ and $a_{i j} \wedge b_{i j}=0$ for all $i, j=1,2,3, \ldots, n$. Put $A^{c}=\left[b_{i j}\right]_{n \times n}$, we get $A \sqcup A^{c}=\top$ and $A \sqcap A^{c}=\perp$. Then $M_{n \times n}(L)$ is complemented.

Conversely, let $M_{n \times n}(L)$ be complemented, i.e., for any $A=\left[a_{i j}\right]_{n \times n} \in M_{n \times n}(L)$, there is $A^{c}=\left[b_{i j}\right]_{n \times n}$ such that $A \sqcup A^{c}=\top$ and $A \sqcap A^{c}=\perp$. Then $a_{i j} \vee b_{i j}=1$ and $a_{i j} \wedge b_{i j}=0$ for all $i, j=1,2,3, \ldots, n$, i.e., $L$ is complemented.

Corollary 31. $\mathbb{M}_{n \times n}(L)$ is Boolean iff $L$ is Boolean.

Let $A=\left[a_{i j}\right]_{n \times n} \in M_{n \times n}(L)$. Define $A^{*}=\left[a_{i j}^{*}\right]_{n \times n}$, where $a_{i j}^{*}=a_{i j} \rightarrow 0$ for all $1 \leq i, j \leq n$. 
Theorem 32. If $A, B \in M_{n \times n}(L)$, we have

1) if $A \preceq B$, then $B^{*} \preceq A^{*}$,

2) $A \preceq A^{* *}, \top^{*}=\perp, \perp^{*}=\top$,

3) $A^{* * *}=A^{*}$,

4) $(A \sqcap B)^{*}=A^{*} \sqcup B^{*},(A \sqcup B)^{*}=A^{*} \sqcap B^{*}$,

5) $(A \sqcap B)^{* *}=A^{* *} \sqcap B^{* *},(A \sqcup B)^{* *}=A^{* *} \sqcup B^{* *}$,

6) $\left(A \triangleright B^{*}\right)=(A \triangleright B)^{*}$.

Theorem 33. Let $C \in B\left(M_{n \times n}(L)\right)$. Then $C^{c}=C^{*}$ and $C^{* *}=C$.

Proof. Let $C \in B\left(M_{n \times n}(L)\right)$. Put $A=C^{c}$, then $C \sqcup A=\top$ and $C \sqcap A=\perp$. Since $C \sqcap A=\perp$, we have $c_{i j} \wedge a_{i j}=0$, for all $1 \leq i, j \leq n$. But $c_{i j} \odot a_{i j} \leq c_{i j} \wedge a_{i j}=0$ implies that $c_{i j} \odot a_{i j}=0$. By adjoint property $a_{i j} \leq c_{i j}^{*}$. Then

$$
\text { (1) } A=C^{c} \preceq C^{*} \text {. }
$$

On the other hand

$$
\begin{aligned}
c_{i j}^{*} & =1 \odot c_{i j}^{*} \\
& =\left(c_{i j} \vee c_{i j}^{\prime}\right) \odot c_{i j}^{*} \\
& =\left(c_{i j} \odot c_{i j}^{*}\right) \vee\left(c_{i j}^{\prime} \odot c_{i j}^{*}\right) \\
& =0 \vee\left(c_{i j}^{\prime} \odot c_{i j}^{*}\right) \\
& =\left(c_{i j}^{\prime} \odot c_{i j}^{*}\right) \leq c_{i j}^{\prime}
\end{aligned}
$$

Then $c_{i j}^{*} \leq c_{i j}^{\prime}$ and it follows that

(2) $C^{*} \preceq C^{c}=A$.

From (1) and (2) we have $C^{*}=C^{c}$ and $C^{* *}=C^{c c}=C$.

Corollary 34. The following conditions are equivalent:

i) $C \in B\left(M_{n \times n}(L)\right)$;

ii) $C \sqcup C^{*}=\top$.

Proof. To prove (ii) from (i), for every $C \in M_{n \times n}(L)$ let $C \in B\left(M_{n \times n}(L)\right)$. Then $C \sqcup C^{c}=C \sqcup C^{*}=\top$, by Theorem 33 ,

Conversely, if $C \sqcup C^{*}=\top$, by Theorem 32 T* $=\perp=\left(C \sqcup C^{*}\right)^{*}=C^{*} \sqcap C^{* *} \succeq$ $C^{*} \sqcap C$. So $C^{*} \sqcap C=\perp$. We get $C \in B\left(M_{n \times n}(L)\right)$.

Proposition 35. Let $L$ be a complete residuated lattice. Then $\mathbb{M}_{n \times n}(L)$ is Brouwerian iff $L$ is Brouwerian. 
Proof.

$$
\begin{aligned}
A \sqcap\left(\sqcup_{k \in K} B_{k}\right)=\sqcup_{k \in K}\left(A \sqcap B_{k}\right) & \Leftrightarrow\left(A \sqcap\left(\sqcup_{k \in K} B_{k}\right)\right)_{i j}=\left(\sqcup_{k \in K}\left(A \sqcap B_{k}\right)\right)_{i j} \\
& \Leftrightarrow\left(a_{i j} \wedge\left(\bigvee_{k \in K} b_{k_{i j}}\right)\right)=\left(\bigvee_{k \in K}\left(a_{i j} \wedge b_{k_{i j}}\right)\right),
\end{aligned}
$$

for all $1 \leq i, j \leq n$.

For $A \in M_{n \times n}(L)$. The powers of $A, A^{n}$ for integers $n \geq 0$ are defined inductively as : $A^{0}=I_{n}$ and $A^{n}=A^{n-1} \square A$.

Definition 36. An element $A \in M_{n \times n}(L)$ is called idempotent iff $A^{2}=A$, and it is called nilpotent if there exists a natural number $n$ such that $A^{n}=\perp$. The least such $n$ is called the nilpotence order of $A$ and is denoted by $\operatorname{ord}(A)$; if there is no such $n$ then $\operatorname{ord}(A)=\infty$.

Theorem 37. $L$ is locally finite, if and only if $M_{n \times n}\left(L^{*}\right)$ is locally finite (every $A \in M_{n \times n}\left(L^{*}\right)$ is nilpotent $)$, where $L^{*}=L \backslash\{1\}$.

Proof. Let $D=\left\{a_{1}, a_{2}, \ldots, a_{k}\right\}$ denote the set of all elements (entries) of $A \in$ $M_{n \times n}\left(L^{*}\right)$. Put $s=\max \left\{t \in \mathbb{N} \mid a_{i}^{t}=0 ;\right.$ forall $\left.1 \leq i \leq k\right\}$. Let $s^{\prime}=s k$. Then $A^{s^{\prime}}=\perp$, because $\odot$ is commutative and if $A^{s k}=\left[a_{i j}\right]_{n \times n}$, then $a_{i j}=$ $\bigvee_{t=1}^{n}\left(a_{i t} \odot a_{t j}\right)$ such that $a_{i t}$ is entries of $A^{s k-1}$. Then $a_{i t}=\bigvee_{t^{\prime}=1}^{n}\left(a_{i t^{\prime}} \odot a_{t^{\prime} t}\right)$ so $a_{i j}=\bigvee_{t=1}^{n}\left(\bigvee_{t^{\prime}=1}^{n}\left(a_{i t^{\prime}} \odot a_{t^{\prime} t}\right) \odot a_{t j}\right)$, where $a_{i t^{\prime}}$ is entries of $A^{s k-2}$ and $a_{t^{\prime} t}$ is entries of $A$. By continue this process we get $a_{i j}=0$.

The converse is trivially true, because for every $a \in L, a \neq 1$, we may consider $A=[a]_{n \times n}$.

For every $A \in M_{n \times n}(L)$, we define $A^{*}=\left[a_{i j}^{*}\right]_{n \times n}$ where $A=\left[a_{i j}\right]_{n \times n}$. Then

Proposition 38. If $L$ is locally finite, then for every $A \in M_{n \times n}(L)$

1) $A^{*}=\perp$ iff $A=\top$,

2) $A^{*}=\top$ iff $A=\perp$,

3) $\perp \prec A \prec \top$ iff $\perp \prec A^{*} \prec \top$.

Proof. Let $A=\left[a_{i j}\right]_{n \times n}$. Then $a_{i j}=1$ iff $a_{i j}^{*}=0$ for all $1 \leq i, j \leq n$, that imply $A^{*}=\perp$ iff $A=\top$. The proof of (2) is similar. For (3), let $\perp \prec A \prec \top$. So there is $a_{i j} \in A$ such that $0<a_{i j}<1$, for some $1 \leq i, j \leq n$. Then by Proposition 13 . $0<a_{i j}^{*}<1$ and we get $\perp \prec A^{*} \prec \top$.

We note that nilpotent matrices over rings are matrices whose diagonal entries are zero, but this is not true in the case of matrices over residuated lattices as the following example shows:

Example 39. Let $L=\{0, a, b, c, 1\}$, where $0<a<b<c<1$ and the operations $\odot, \rightarrow$ given by the following tables. Then $L=(L, \vee, \wedge, \odot, \rightarrow, 0,1)$ is a commutative 
residuated lattice and we have: $\operatorname{ord}(0)=1, \operatorname{ord}(a)=2, \operatorname{ord}(b)=2, \operatorname{ord}(c)=3$. Thus, $L$ is a locally finite commutative residuated lattice [4].

\begin{tabular}{|c|c|c|c|c|c|c|c|c|c|c|c|}
\hline$\odot$ & 0 & $a$ & $b$ & $c$ & 1 & $\rightarrow$ & 0 & $a$ & $b$ & $c$ & 1 \\
\hline 0 & 0 & 0 & 0 & 0 & 0 & 0 & 1 & 1 & 1 & 1 & 1 \\
\hline$a$ & 0 & 0 & 0 & 0 & $a$ & $a$ & $c$ & 1 & 1 & $d$ & 1 \\
\hline$b$ & 0 & 0 & 0 & 0 & $b$ & $b$ & $b$ & $c$ & 1 & 1 & 1 \\
\hline$c$ & 0 & 0 & $a$ & $a$ & $c$ & $c$ & $b$ & $c$ & $c$ & 1 & 1 \\
\hline 1 & 0 & $a$ & $b$ & $c$ & 1 & 1 & 0 & $a$ & $b$ & $c$ & 1 \\
\hline
\end{tabular}

Then $A=\left(\begin{array}{cc}a & 0 \\ 0 & b\end{array}\right), B=\left(\begin{array}{cc}a & c \\ 0 & b\end{array}\right) \in M_{2 \times 2}(L)$ are two nilpotent matrices, because $A^{2}=B^{3}=\perp$.

It is well known that filters and ideals play important roles in algebras specially in defining congruence relations and corresponding quotient algebras. This motivates us to define ideals, filters of $M_{n \times n}(L)$. We give some examples and establish some relations between these subsets of $M_{n \times n}(L)$ and the corresponding ones in L. We will employ these properties to characterize invertible matrices in $M_{n \times n}(L)$.

Definition 40. A nonempty subset $S$ of $M_{n \times n}(L)$ is called a filter of $M_{n \times n}(L)$ if the following conditions hold:

1) if $A, B \in S$, then $A \boxminus B \in S$,

2) if $A \in S, B \in M_{n \times n}(L)$ and $A \preceq B$ then $B \in S$.

A filter $S$ of $M_{n \times n}(L)$ is proper if $S \neq M_{n \times n}(L)$. Clearly, $S$ is a proper filter iff $\perp \notin S$.

Example 41. Let $L$ be a residuated lattice defined in Example 25. Then $S=$ $\left\{\left(\begin{array}{ll}1 & a \\ 1 & 1\end{array}\right),\left(\begin{array}{ll}1 & b \\ 1 & 1\end{array}\right),\left(\begin{array}{ll}1 & 1 \\ 1 & 1\end{array}\right)\right\}$, is a proper filter of $M_{2 \times 2}(L)$.

Theorem 42. $M_{n \times n}(F)$ for $F \subseteq L$ is a filter of $M_{n \times n}(L)$ iff $F$ is a filter of $L$.

Proof. Let $F$ be a filter of $L$. We prove that conditions (1) and (2) of Definition 40 hold. For(1) let $A, B \in M_{n \times n}(F)$. Take $A=\left[a_{i j}\right]_{n \times n}, B=\left[b_{i j}\right]_{n \times n}$ for all $1 \leq i, j \leq n$ and $a_{i j}, b_{i j} \in F$, but $F$ is a filter of $L$, then $a_{i t} \odot b_{t j} \in F$ for all $1 \leq i, t \leq n$. On the other hand we know that $a_{i t} \odot b_{t j} \leq \bigvee_{t=1}^{n} a_{i t} \odot b_{t j}=(A \odot B)_{i j} \in$ $F$. So $A \square B \in M_{n \times n}(F)$. For (2) let $A \preceq B$ and $A=\left[a_{i j}\right]_{n \times n} \in M_{n \times n}(F)$ but because we assume that $\mathrm{F}$ is a filter and $a_{i j} \leq b_{i j}$ we have $b_{i j} \in F$, then $B=\left[b_{i j}\right]_{n \times n} \in M_{n \times n}(F)$.

Conversely, let $M_{n \times n}(F)$ for $F \subseteq L$ be a filter of $M_{n \times n}(L)$, we show that $F$ is a filter of $L$, i.e., the conditions (i) and (ii) of Definition 3 is hold . For (i) let $a, b \in F$ and put $A=[a]_{n \times n}, B=[b]_{n \times n}$. Then $A, B \in M_{n \times n}(F)$ so $A \odot B=[a \odot b]_{n \times n} \in$ $M_{n \times n}(F)$, then $a \odot b \in F$. Finally to prove (ii) let $a \in F, b \in L$ and $a \leq b$. We set $A=[a]_{n \times n}, B=[b]_{n \times n} \in M_{n \times n}(L)$, clear that $A \preceq B$, but $M_{n \times n}(F)$ is a filter of $M_{n \times n}(L)$ then $B \in M_{n \times n}(F)$, i.e., $b \in F$. 
Corollary 43. $M_{n \times n}(F)$ for $F \subseteq L$ is a proper filter of $M_{n \times n}(L)$ iff $F$ is a proper filter of $L$.

Proposition 44. Let $F$ be a filter of $L$. Then:

1) $\top \in M_{n \times n}(F)$,

2) If $A, B \in M_{n \times n}(F)$, then $A \sqcap B \in M_{n \times n}(F)$,

3) If $A, A \triangleright B(A, A \triangleleft B) \in M_{n \times n}(F)$, then $B \in M_{n \times n}(F)$.

Proof. (1) holds because $1 \in F$. For (2) let $A=\left[a_{i j}\right]_{n \times n}, B=\left[b_{i j}\right]_{n \times n} \in M_{n \times n}(F)$. Then $a_{i j}, b_{i j} \in F$, but $F$ is a filter of $L$, then $a_{i j} \odot b_{i j} \leq a_{i j} \wedge b_{i j} \in F$. So $A \sqcap B \in M_{n \times n}(F)$. To prove (3), we know that $(A \triangleright B)_{i j}=\bigwedge_{t=1}^{n}\left(a_{j t} \rightarrow b_{i t}\right) \in F$ $1 \leq i, j \leq n$. Recall that if $a \in F$ and $a \rightarrow b \in F$ imply that $b \in F$ because $a \odot(a \rightarrow b) \leq b$. Now $a_{j t} \rightarrow b_{i t} \geq \bigwedge_{t=1}^{n}\left(a_{j t} \rightarrow b_{i t}\right) \in F$ implies that $a_{j t} \rightarrow b_{i t} \in F$ for every $1 \leq i, j, t \leq n$ and from above it follows that $b_{i t} \in F$ for $1 \leq i, t \leq n$. Then $B \in M_{n \times n}(F)$. The proof of another case is similar.

Corollary 45. If $F_{1}, F_{2}$ are two filters of $L$ such that $F_{1} \cap F_{2}=\{1\}$, then for every $A \in M_{n \times n}\left(F_{1}\right)$ and $B \in M_{n \times n}\left(F_{2}\right)$ such that $A \neq \top \neq B$, we have $A \triangleright B(A \triangleleft B) \notin$ $M_{n \times n}\left(F_{1}\right)$ and $B \triangleright A(B \triangleleft A) \notin M_{n \times n}\left(F_{2}\right)$.

Remark 46. The Proposition 44 does not hold for every filter of $M_{n \times n}(L)$. For instance, in Example 41 . if we set $A=\left(\begin{array}{ll}1 & a \\ 1 & 1\end{array}\right)$ and $B=\left(\begin{array}{ll}1 & b \\ 1 & 1\end{array}\right)$. Then $A \sqcap B=\left(\begin{array}{cc}1 & 0 \\ 1 & 1\end{array}\right) \notin S$.

Lemma 47. If $F_{i}$ are filters of $M_{n \times n}(L)$, then $\bigcap_{i \in I} F_{i}$ is a filter of $M_{n \times n}(L)$.

Proposition 48. If $L$ is a residuated lattice, then the sets $M_{n \times n}(L)^{-}=\{A \in$ $\left.M_{n \times n}(L) \mid A^{-}=\perp\right\}$ and $M_{n \times n}(L)^{\sim}=\left\{A \in M_{n \times n}(L) \mid A^{\sim}=\perp\right\}$ are proper filters of $M_{n \times n}(L)$.

Proof. If $A, B \in M_{n \times n}(L)^{-}$, then by Proposition 22 , we have $(A \sqcup B)^{-}=(A \triangleright$ $\left.B^{-}\right)=(A \triangleright \perp)=\perp$. So $A \square B \in M_{n \times n}(L)^{-}$. If $A \in M_{n \times n}(L)^{-}, B \in M_{n \times n}(L)$ such that $A \preceq B$, then by Proposition 19, $B^{-} \preceq A^{-}=\perp$. So $B^{-}=\perp$, and $B \in M_{n \times n}(L)^{-}$. But by Corollary $21, \perp \notin M_{n \times n}(L)^{-}$, and we conclude that $M_{n \times n}(L)^{-}$is a proper filter of $M_{n \times n}(L)$. Similarly for the case of $M_{n \times n}(L)^{\sim}$.

Definition 49. A subset $S \subseteq M_{n \times n}(L)$ is called an ideal of $M_{n \times n}(L)$ if the following conditions hold:

i) $\perp \in S$,

ii) if $A, B \in S$, then $A \sqcup B \in S$,

iii) if $A \in M_{n \times n}(L), B \in S$ and $A \preceq B$, then $A \in S$.

An ideal $S$ of $M_{n \times n}(L)$ is called proper if $S \neq M_{n \times n}(L)$. Clearly, $S$ is proper iff $\top \notin S$. The ideal $S$ of $M_{n \times n}(L)$ is called prime if for all $A, B \in M_{n \times n}(L)$ such that $A \sqcap B \in S$, then $A \in S$ or $B \in S$. 
Theorem 50. $M_{n \times n}(I)$ for $I \subseteq L$ is an ideal of $M_{n \times n}(L)$ iff $I$ is an ideal of $L$.

Corollary 51. For ideal $I$ of $L, M_{n \times n}(I)$ is proper iff $I$ is proper.

Definition 52. For $X \subseteq M_{n \times n}(L), B \in M_{n \times n}(L)$, we define $[X, B]=\left\{A \in M_{n \times n}(L) \mid A \sqcup B \in X\right\}$, in particular if $X=\{\perp\}$, then $[\{\perp\}, B]$ is called an annihilator of the matrix $B$. Generally for $X, Y \subseteq M_{n \times n}(L)$, we define

$$
[X, Y]=\left\{A \in M_{n \times n}(L) \mid A \boxminus B \in X ; \text { for all } B \in Y\right\} \text {. }
$$

Theorem 53. For subsets $X, Y \subseteq M_{n \times n}(L)$, if $X$ is an ideal of $M_{n \times n}(L)$, then $[X, Y]$ is an ideal of $M_{n \times n}(L)$.

Proof. If $X$ is an ideal of $M_{n \times n}(L)$, then $\perp \in X$, and we know that $\perp \square B=\perp$ for all $B \in Y$, so $\perp \in[X, Y]$. We assume that $A, A^{\prime} \in[X, Y]$, then for all $B \in Y$ we have $A \boxminus B, A^{\prime} \square B \in X$. But $X$ is an ideal of $M_{n \times n}(L)$, so $(A \boxminus B) \sqcup\left(A^{\prime} \boxminus B\right)=$ $\left(A \sqcup A^{\prime}\right) \triangleright B \in X$. Therefore $A \sqcup A^{\prime} \in[X, Y]$. On the other hand if we put $A \preceq A^{\prime}$ and $A^{\prime} \in[X, Y]$, then for all $B \in Y$ we have $A^{\prime} \boxminus B \in X$. But since $X$ is an ideal and $A \sqcup B \preceq A^{\prime} \bullet B$, we get $A \in[X, Y]$. Then $[X, Y]$ is an ideal of $M_{n \times n}(L)$.

Corollary 54. For every $B \in M_{n \times n}(L),[\{\perp\}, B]$ is an ideal of $M_{n \times n}(L)$.

Theorem 55. Let $L$ a Godel residuated lattice. If $X$ is a prime ideal of $M_{n \times n}(L)$, then $[X, Y]$ is a prime ideal, for every $Y \subseteq M_{n \times n}(L)$.

Proof. Let $A, B \in M_{n \times n}(L)$ be arbitrary elements such that $A \sqcap B \in[X, Y]$ and $A, B \notin[X, Y]$. Since $L$ is Godel, then by using Theorem 2, for every $D \in Y$, $(A \sqcap B) \square D=(A \sqcup D) \sqcap(B \sqcup D) \in X$. But $X$ is a prime ideal. Then $A \boxminus D \in X$ or $B \boxminus D \in X$ for every $D \in Y$, and we get $A \in[X, Y]$ or $B \in[X, Y]$, which contradicts $A, B \notin[X, Y]$.

Lemma 56. Let $X, X_{1}, X_{2}, Y, Y_{1}, Y_{2}$ and $X_{i}, Y_{i}, i \in I$, be arbitrary subsets of $M_{n \times n}(L)$. Then

1) If $X_{1} \subseteq X_{2}$ then $\left[X_{1}, Y\right] \subseteq\left[X_{2}, Y\right]$,

2) If $Y_{1} \subseteq Y_{2}$ then $\left[X, Y_{2}\right] \subseteq\left[X, Y_{1}\right]$,

3) If $Y=\phi$ then $[X, Y]=M_{n \times n}(L)$,

4) If $X=\phi, Y \neq \phi$ then $[X, Y]=\phi$,

5) If $Y=\left\{I_{n}\right\}$, then $[X, Y]=X$,

6) $[\{\perp\},\{\perp\}]=M_{n \times n}(L),[\{\top\},\{\top\}]=\left\{\top, I_{n}\right\}$. 


$$
\begin{aligned}
& \text { 7) }[X, Y]=\bigcap_{B \in Y}[X, B], \\
& \text { 8) }\left[X, M_{n \times n}(L)\right] \subseteq X, \\
& \text { 9) }\left[X, M_{n \times n}(L)\right]=\phi \text { iff } \perp \notin X, \\
& \text { 10) }[X, \perp]=M_{n \times n}(L) \text { iff } \perp \in X, \\
& \text { 11) }\left(\left[X_{1}, Y_{1}\right] \cap\left[X_{2}, Y_{2}\right]\right) \subseteq\left[X_{1} \cap X_{2}, Y_{1} \cap Y_{2}\right] \\
& \text { 12) }\left[X, \bigcup_{i \in I} Y_{i}\right]=\bigcap_{i \in I}\left[X, Y_{i}\right] \subseteq \bigcup_{i \in I}\left[X, Y_{i}\right] \subseteq\left[X, \bigcap_{i \in I} Y_{i}\right], \\
& \text { 13) } \bigcap_{i \in I}\left[X_{i}, Y\right]=\left[\bigcap_{i \in I} X_{i}, Y\right] \text { and } \bigcup_{i \in I}\left[X_{i}, Y\right] \subseteq\left[\bigcup_{i \in I} X_{i}, Y\right]
\end{aligned}
$$

Proof. (1) Let $A \in\left[X_{1}, Y\right]$ be an arbitrary matrix. Then for every $B \in Y$, $A \boxminus B \in X_{1}$. On the other hand $X_{1} \subseteq X_{2}$, therefore for every $B \in Y, A \boxminus B \in X_{2}$. Then $A \in\left[X_{2}, Y\right]$. (2) is proved similarly.

(3) If $Y=\phi$, then $[X, \phi]=\left\{A \in M_{n \times n}(L) \mid A \boxminus B \in X\right.$; for all $\left.B \in \phi\right\}=M_{n \times n}(L)$.

(4) is proved similarly.

(5) If $Y=I_{n}$, then $\left[X,\left\{I_{n}\right\}\right]=\left\{A \in M_{n \times n}(L) \mid A \boxminus I_{n}=A \in X\right\}=M_{n \times n}(L) \cap$ $X=X$. (6) proved similarly.

(7) $A \in[X, Y]$ iff for all $B \in Y, A \boxminus B \in X$ iff for every $B \in Y, A \in[X,\{B\}]$ iff $A \in \bigcap_{B \in Y}[X,\{B\}]$.

(8) Let $A$ be an arbitrary element of $\left[X, M_{n \times n}(L)\right]$. Then for all $B \in M_{n \times n}(L)$, $A \boxminus B \in X$. Therefore $A \boxminus I_{n}=A \in X$. So $\left[X, M_{n \times n}(L)\right] \subseteq X$.

(9) Let $\left[X, M_{n \times n}(L)\right] \neq \phi$. Then there is $A \in\left[X, M_{n \times n}(L)\right]$ and we have $A \boxminus \perp=$ $\perp \in X$, which contradicts $\perp \notin X$. Conversely, let $\perp \in X$. Then for every $A \in M_{n \times n}(L)$ we have $\perp \boxminus A=\perp \in X$. Therefore $\perp \in\left[X, M_{n \times n}(L)\right]$ which contradicts $\left[X, M_{n \times n}(L)\right]=\phi$. So $\perp \notin X$.

(10) Let $\perp \in X$ then $[X,\{\perp\}]=M_{n \times n}(L)$. Conversely, let $\perp \notin X$, then $[X,\{\perp\}]=$ $\phi$, which contradicts $[X,\{\perp\}]=M_{n \times n}(L)$.

(11) Let $A \in\left(\left[X_{1}, Y_{1}\right] \cap\left[X_{2}, Y_{2}\right]\right)$ be an arbitrary element. Then for every $B \in Y_{1}$ and $C \in Y_{2}, A \boxminus B \in X_{1}$ and $A \boxminus C \in X_{2}$. Since $Y_{1} \cap Y_{2} \subseteq Y_{1}, Y_{2}$, for every $D \in Y_{1} \cap Y_{2}$, we have $A \boxminus D \in X_{1} \cap X_{2}$. So $A \in\left[X_{1} \cap X_{2}, Y_{1} \cap Y_{2}\right]$.

(12) For every $i \in I, Y_{i} \subseteq \bigcup_{i \in I} Y_{i}$. Then by (2) we have for every $i \in I$, $\left[X, \bigcup_{i \in I} Y_{i}\right] \subseteq\left[X, Y_{i}\right]$. Therefore for every $i \in I,\left[X, \bigcup_{i \in I} Y_{i}\right]$ is a lower bound for $\left[X, Y_{i}\right]$. Then $\left[X, \bigcup_{i \in I} Y_{i}\right] \subseteq \bigcap_{i \in I}\left[X, Y_{i}\right]$. Conversely, let $A \in \bigcap_{i \in I}\left[X, Y_{i}\right]$ be an arbitrary element. Then for every $i \in I, B_{i} \in Y_{i}, A \boxminus B_{i} \in X$. Now we let $C \in \bigcup_{i \in I} Y_{i}$ be an arbitrary element. Then there is $j \in I$, such that $C \in Y_{j}$. So $A \boxminus C \in X$ and we get $A \in\left[X, \bigcup_{i \in I} Y_{i}\right]$.

Again by (2), for every $i \in I,\left[X, Y_{i}\right] \subseteq\left[X, \bigcap_{i \in I} Y_{i}\right]$. Then for every $i \in I$, $\left[X, \bigcap_{i \in I} Y_{i}\right]$, is an upper bound for every $\left[X, Y_{i}\right]$. Therefore $\bigcup_{i \in I}\left[X, Y_{i}\right] \subseteq\left[X, \bigcap_{i \in I} Y_{i}\right]$.

(13) For every $i \in I, \bigcap_{i \in I} X_{i} \subseteq X_{i}$. Then by (1) we have for every $i \in I$, 
$\left[\bigcap_{i \in I} X_{i}, Y\right] \subseteq\left[X_{i}, Y\right]$. Then $\left[\bigcap_{i \in I} X_{i}, Y\right] \subseteq \bigcap_{i \in I}\left[X_{i}, Y\right]$. Conversely let $A \in$ $\bigcap_{i \in I}\left[X_{i}, Y\right]$ be an arbitrary element. Then for every $i \in I, B \in Y, A \boxminus B \in X_{i}$. Therefore for every $B \in Y, A \boxminus B \in \bigcap_{i \in I} X_{i}$, so $A \in\left[\bigcap_{i \in I} X_{i}, Y\right]$. Similarly we can prove that $\bigcup_{i \in I}\left[X_{i}, Y\right] \subseteq\left[\bigcup_{i \in I} X_{i}, Y\right]$.

Remark 57. The converse of 4,5 and identities, in 8 and 11 are not necessarily true, because for (4) if we let $X=\{\top\}, Y=\{\perp\}$. Then $[X, Y]=\phi, Y \neq \phi$ but $X \neq \phi$.

For (5) set $X=M_{n \times n}(L), Y=\{\perp\}$. Then $[X, Y]=X$, and $Y \neq\left\{I_{n}\right\}$.

For (8) put $X=\left\{\perp, I_{n}, \top\right\}$. Then, $\left[X, M_{n \times n}(L)\right]=\{\perp\} \subset\left\{\perp, I_{n}, \top\right\}=X$.

For (11) it is enough to put $X_{1}=\left\{\perp, I_{n}, \top\right\}, X_{2}=\left\{\perp, I_{n}\right\}, Y_{1}=M_{n \times n}(L)$ and $Y_{2}=\{\perp\}$. Then $\left[X_{1}, Y_{1}\right]=\{\perp\},\left[X_{2}, Y_{2}\right]=M_{n \times n}(L)$. So $\left(\left[X_{1}, Y_{1}\right] \cap\left[X_{2}, Y_{2}\right]\right)=$ $\{\perp\} \subset\left[X_{1} \cap X_{2}, Y_{1} \cap Y_{2}\right]=\left[X_{2}, Y_{2}\right]=M_{n \times n}(L)$.

\section{Conclusion}

We introduced the algebra of square matrices over a commutative residuated lattice L and investigated some properties of this structure. We showed that this algebra behaves like a residuated lattice, but there are some differences. We explored a set of like and unlike properties of $M_{n \times n}(L)$. Also, in this paper we studied relationship between the residuated lattice $L$ and $M_{n \times n}(L)$. In the next paper, we discuss the notion of invertibility of matrices. We give necessary and sufficient conditions on matrices to be invertible. We use invertible matrices for solving relational equations over residuated lattice. In fact our main goal is solving the system of equations with coefficients from residuated lattices. As noted we need to know the algebraic structure of matrices over residuated lattices.

Acknowledgement. The authors would like to express their special thanks to the referees for the comments that greatly improved our manuscript.

\section{REFERENCES}

[1] Adak, A. K., Bhowmik, M. and Pal, M., Some properties of generalized intuitionistic fuzzy nilpotent matrices over distributive lattices, Fuzzy Inf. Eng, 4 (2012), 371-387.

[2] Atanassov, K., intuitionistic fuzzy sets, in V.Sgurev, ed.,Vii ITKRS Session, Sofia, Tune 1983.

[3] Belohlavek, R., Concept lattices and order in fuzzy logic. emphAnn. Pure Appl. Logic, 128(3) (2004), 277-298.

[4] Ciungu, L. C, Classes of residuated lattices, Annals of University of Craiova, Math. Comp, Sci. Ser., 33 (2006), 189-207.

[5] Galatos, N., Jipsen, P., Kowalski, T. and Ono, H., Residuated lattices: An Algebraic Gelimpse at Substructural Logics, Volume 151 of studies in Logic and the Foundations of Mathematics, Elsevier, Amesterdam, 2007.

[6] Ganter, B. and Wille, R., Formal Concepts Analysis, Mathematical Foundations. Springer, Berlin, 1990.

[7] Hosseinyazdi, M., The optimization problem over a distributive lattice, Journal of Global Optimization, 41 (2008), 283-298. 
[8] Kim K. H. and Roush, F. W., Generalized fuzzy matrices, Fuzzy sets and systems, 4 (1980), 243-315.

[9] Pal, M., Intuitionistic fuzzy determinat, V.U.J. physical sciences, 7 (2001), 87-93.

[10] Pal, M., Khan, S. K. and Shyamal, A. K., Intuitionistic fuzzy matrices. Notes on Intuitionistic fuzzy sets, 8(2) (2002), 51- 62.

[11] Piciu, D., Algebras of Fuzzy Logic, Universitaria din Craiova, 2007.

[12] Sriram S. and Murugadas, P., On semiring of intuitionistic fuzzy matrices, Applied Mathematical. Sciences, 4(23) ( 2010), 1099-1105.

[13] Thomason, M. G., Convergence of powers of a fuzzy matrix, J. Math. Anal. Appl, 57 (1977), 476-486.

[14] Wilding, D., Linear algebra over semiring, university of Manchester, 2014.

[15] Wille. R., Restructuring lattice theory: An approach based on concepts. In I. Rival, editor, Ordered sets, volume 83 of NATO Advanced Study Institute Series, pages 445- 470, Springer, Dordrecht, 1982

[16] Xiao, Y., Xue, T., Xue, Z. and Cheng, H., A new kind of intuitionistic fuzzy implication, Journal of Information and Computational Sciences, 8(13) (2011), 2839-2849.

Current address: S. Balaei: Department of Pure Mathematics, Faculty of Mathematics and Computer, Shahid Bahonar University of Kerman, Kerman, Iran.

E-mail address: s.balaei@uk.ac.ir

ORCID Address: http://orcid.org/0000-0002-0861-0781

Current address: E. Eslami: Department of Pure Mathematics, Faculty of Mathematics and Computer, Shahid Bahonar University of Kerman, Kerman, Iran.

E-mail address: es.eslami@uk.ac.ir

ORCID Address: http://orcid.org/0000-0001-9353-8763

Current address: A. Borumand Saeid: Department of Pure Mathematics, Faculty of Mathematics and Computer, Shahid Bahonar University of Kerman, Kerman, Iran.

E-mail address: arsham@uk.ac.ir

ORCID Address: http://orcid.org/0000-0001-9495-6027 